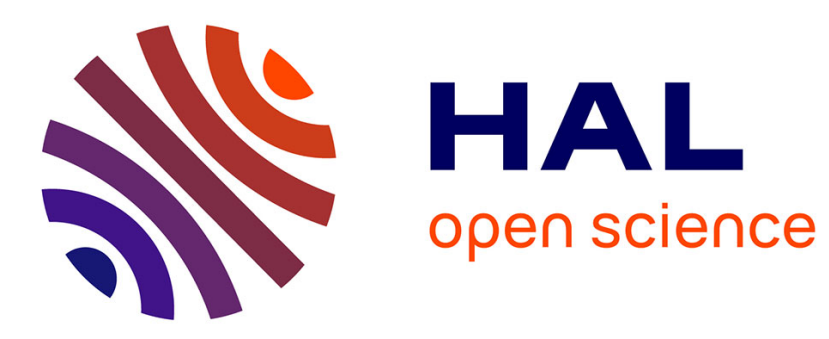

\title{
Optimized butterfly-based lifting scheme for semi-regular meshes
}

Aymen Kammoun, Frédéric Payan, Marc Antonini

\section{To cite this version:}

Aymen Kammoun, Frédéric Payan, Marc Antonini. Optimized butterfly-based lifting scheme for semi-regular meshes. IEEE International Conference in Image Processing (ICIP), Sep 2011, Brussels, Belgium. pp.1269-1272. hal-00588168

\section{HAL Id: hal-00588168 https://hal.science/hal-00588168}

Submitted on 22 Apr 2011

HAL is a multi-disciplinary open access archive for the deposit and dissemination of scientific research documents, whether they are published or not. The documents may come from teaching and research institutions in France or abroad, or from public or private research centers.
L'archive ouverte pluridisciplinaire HAL, est destinée au dépôt et à la diffusion de documents scientifiques de niveau recherche, publiés ou non, émanant des établissements d'enseignement et de recherche français ou étrangers, des laboratoires publics ou privés. 


\title{
OPTIMIZED BUTTERFLY-BASED LIFTING SCHEME FOR SEMI-REGULAR MESHES
}

\author{
Aymen Kammoun, Frédéric Payan, Marc Antonini, IEEE Member \\ Laboratoire I3S (UMR 6070 CNRS-Université de Nice - Sophia Antipolis) \\ 2000, Route des Lucioles - 06903 Sophia Antipolis - France
}

\begin{abstract}
In this paper, we propose an optimization of the lifted Butterfly scheme for semi-regular meshes. This optimization consists in adapting the predict and update steps at each level of resolution for a given semi-regular mesh. The motivation is the improvement of the multiresolution analysis in order to increase the compression performances of the subsequent geometry coder. We first compute an optimized prediction scheme that minimizes the L1-norm of the wavelet coefficients for each level of resolution, independently. We then compute the update scheme in order to preserve the data average $\left(0^{t h}\right.$ moment $)$ at the lower resolution. Experimental results shows that our technique globally reduces the entropy of the wavelet coefficients of any semi-regular mesh. Consequently our contribution also improves the compression performances of the zerotree coder $P G C$.
\end{abstract}

Index Terms - Wavelets, Butterfly scheme, Compression, Semi-regular mesh

\section{INTRODUCTION}

Today triangular meshes can be defined by several millions of vertices, and more. A simple representation of these densely sampled meshes is consequently huge. Compression is one relevant tool to allow compact storage and/or fast transmission in bandwidth-limited applications of such massive data, and many techniques have been already proposed [1]. To our knowledge, wavelet-based coders that take semi-regular meshes as input are the most efficient [2, 3, 4].

Many works have been done to improve the wavelet construction for surface meshes. Dahlke et al. first introduced the construction of wavelets on the sphere, by using a tensor product basis where one factor is an exponential spline [5]. Then, a continuous wavelet transform on the sphere and its semi-discretization were proposed in [6]. In 1995, Schröder et al. proposed the lifting scheme, a simple technique for constructing biorthogonal wavelets on the sphere with customized properties [7]. In 1997, Lounsbery et al. proposed new wavelets for surface meshes, based on subdivision surfaces [8]. In 2000, Kovacevic et al. proposed a wavelet transform for meshes that relies on the lifting scheme and interpolating scaling functions [9]. In 2004, Bertram proposed a biorthogonal wavelet construction based on loop [10], whereas Li et al. proposed an unlifted Loopbased scheme [11]. In the same time, Valette et al. developed specific wavelets for irregular meshes unlike all other methods [12]. The interpolating Butterfly scheme [13] has been also used for developing wavelets implemented in lifting scheme. The interest of this scheme in multiresolution analysis has been particularly highlighted in compression $[3,4,14]$.

In this paper, we propose an optimized construction of the Butterfly-based lifting scheme $[7,15]$. The idea is to adapt the two main stages of the lifting scheme (prediction and update) to the geometrical characteristics of the input semi-regular mesh. Our motivation is to maximize the sparsity of the wavelet coefficients, and consequently the compression performances of the subsequent geometry coders.

The rest of this paper is organized as follows. Section 2 briefly introduces the lifting scheme for semi-regular meshes. Section 3 presents our optimization technique for the Butterfly-based lifting scheme. Section 4 shows some experimental results and we finally conclude in section 5 .

\section{LIFTING SCHEME FOR SEMI-REGULAR MESHES}

Wavelet transform consists in decomposing a given mesh into a base mesh (low frequency signal) and several sets of wavelet coefficients (high frequency details). Here we only focus on wavelets for semiregular meshes. A semi-regular mesh is based on a mesh hierarchy that represents a given surface at different levels of resolution. The hierarchy exploits the subdivision connectivity to get the different resolutions. Figure 1 shows an example.

Let us note $M^{N}$ a semi-regular mesh defined by $N$ levels of resolution. The geometry of $M^{N}$ is defined by a set of $V^{N}$ vertices $\left\{v_{k}^{N}, k=1 . . V^{N}\right\}$, where $v_{k}^{N}$ is defined by its position in the Euclidean space (three coordinates $x, y$ and $z$ ). Applying a wavelet transform to $M^{N}$ results in one mesh $M^{N-1}$ of lower resolution, and a set of wavelet coefficients $C^{N}=\left\{c_{k}^{N}\right\}$, with $c_{k}^{N}$ a threedimensional coefficient expressed in a local frame. Figure 2 illustrates this wavelet decomposition.

Wavelet transforms are often implemented in lifting scheme [7, 15], which is reversible and faster than implementation based on filter banks.

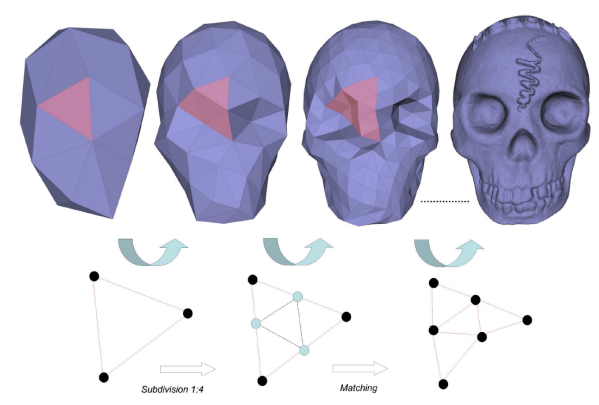

Fig. 1. Up: a semi-regular mesh, from the lowest to the highest resolution. Bottom: the connectivity subdivision.

In our context, this scheme (Figure 3 ) is characterized by:

- a splitting step that divides the vertices of $M^{j}$ in two subsets $M_{0}^{j}$ and $M_{1}^{j}$ (the green and red dots on the figure 2); 


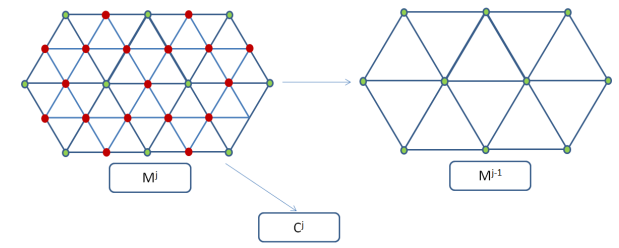

Fig. 2. Wavelet decomposition of a semi-regular mesh. $M^{j}$ is transformed into the "low frequency" mesh $M^{j-1}$ and a set of coefficients $C^{j}$ (associated to the red vertices removed during the decomposition).

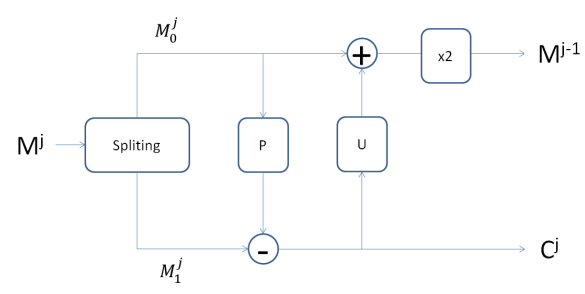

Fig. 3. Lifting scheme for semi-regular meshes.

- a predict step $P$ that takes as input $M_{0}^{j}$ (green dots) and predict the positions of the vertices belonging to $M_{1}^{j}$ (red dots). The prediction errors corresponds to the wavelet coefficients $\left\{c_{k}^{j}\right\}$;

- an update operator $U$ that takes as input $C^{j}$ and modifies the positions of vertices belonging $M_{0}^{j}$. A gain ( $\left.x 2\right)$ is finally applied on $M_{0}^{j}$ to finally obtain the "low frequency" mesh $M^{j-1}$

\section{PROPOSED OPTIMIZATION}

The objective of our works is to optimize the predict $(P)$ and update $(U)$ steps of the Butterfly-based wavelets, in order to improve the efficiency of the subsequent quantizer. Our optimization consists in computing new weights for the stencils of $P$ and $U$. This optimization is done at each level of decomposition during the analysis, producing $P$ and $U$ optimized for each level of resolution.

\subsection{Optimization of the prediction scheme}

For the predict step, we start from the modified Butterfly stencils given by Figure 4 [13]. We apply those stencils on the vertices of $M_{1}^{j}$ to predict their position from the neighbor vertices of $M_{1}^{j}$. Those stencils are given by figure 4 . There are two stencils, a regular and an irregular. The regular stencil (Figure 4(a)) is used when a given vertex has two neighbors (belonging to the lower resolution) having a valence 6 . The irregular stencil is for the other case, i.e. at least one neighbor is irregular (Figure 4(b)). Note that we do not optimize the irregular case, because there are a lot of different configurations for this stencil, since it depends on the valence of the neighbor vertices. Finally, optimizing the different irregular stencils should lead to the computation and the transmission of a too large quantity of side information (to reconstruct the meshes during compression). Instead, we prefer optimizing only the regular case, and using the usual weights given in [13] for the irregular case.

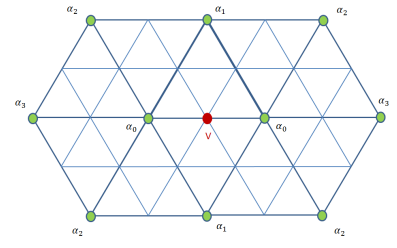

(a) Regular stencil.

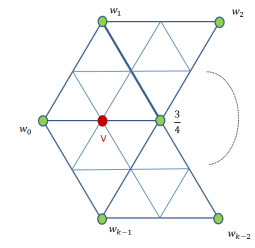

(b) Irregular stencil.
Fig. 4. Stencils of the Butterfly-based prediction $P$.

Considering the resolution $j$, the set of wavelet coefficients $C^{j}$ is computed by using the equation

$$
C^{j}=M_{1}^{j}-P * M_{0}^{j} .
$$

As the majority of wavelet-based coders for semi-regular meshes, we consider separately the tangential components (corresponding to the coordinates $x$ and $y$ of the coefficients) and the normal components (corresponding to the coordinates $z$ ). Hence, we propose to compute i two optimized prediction schemes $P_{x y}^{j}$ and $P_{z}^{j}$ independently, according to the tangential and the normal components, respectively. The same algorithm is currently developed for those two prediction schemes. For clarity, we use $P^{j}$ hereinafter for both $P_{x y}^{j}$ and $P_{z}^{j}$.

In order to maximize the coding performances, we want $C^{j}$ to become as sparse as possible. For this, we choose to minimize the L1-norm of the sets of coefficients $C^{j}$ at each resolution [16]. Finally, maximizing the sparsity of the coefficients amounts to solve the following minimization problem

$$
\min _{\left\{\alpha_{i}^{j}\right\}} \| M_{1}^{j}-P^{j}\left(\left\{\alpha_{i}^{j}\right\} * M_{0}^{j} \|_{1},\right.
$$

where $\left\{\alpha_{i}^{j}\right\}$ represents the weights relative to the prediction scheme $P^{j}$ (see Figure 4(a)).

We resolve this system by using a sequential quadratic programming method and under the constraint: $\sum \alpha_{i}^{j}=1$ (in order to obtain a predictor with a unitary gain).

\subsection{Computation of the new update scheme}

If the prediction $P$ is based on a subdivision scheme, the update $U$ has to be chosen such as to preserve higher order moments [17]. Since we have modified the weights of the prediction scheme $P$ with our optimization technique, we have to compute a new update scheme. For the update step, we still start from the stencils proposed in [13], and given by Figure 5. The coefficient $\beta$ of this stencil depends on the valence $K$ of the given vertex $v$ :

$$
\beta=\frac{\gamma}{K},
$$

where $\gamma$ is usually equal to $3 / 4$.

Considering a given resolution $j$, the low frequency signal $M^{j-1}$ is computed according to

$$
M^{j-1}=2 \times\left(M_{0}^{j}+U^{j} * C^{j}\right) .
$$

So for a given vertex $v_{k}^{j-1}$ of $M^{j-1}$, we can write

$$
v_{k}^{j-1}=2 \times\left(v_{k}^{j}+\sum_{i=1 . . K_{k}^{j}} \frac{\gamma^{j}}{K_{k}^{j}} c_{S_{k}^{j}(i)}^{j}\right),
$$


where $K_{k}^{j}$ is the number of neighbors of $v_{k}^{j}$, and $S_{k}^{j}(i)$ is the set of neighbor vertices of $v_{k}^{j}$.

we propose to compute $\gamma$ in order to preserve the average $\left(0^{t h}\right.$ moment) between $M^{j}$ and $M^{j-1}$ [17]:

$$
\frac{1}{V^{j-1}} \sum_{k=1 . . V^{j-1}} v_{k}^{j-1}=\frac{1}{V^{j}} \sum_{l=1 . . V^{j}} v_{l}^{j},
$$

By combining equations (5) and (6), we obtain

$$
\frac{2}{V^{j-1}} \sum_{k=1 . . V^{j-1}}\left(v_{k}^{j}+\sum_{i=1 . . K_{k}^{j}} \frac{\gamma^{j}}{K_{k}^{j}} c_{S_{k}^{j}(i)}^{j}\right)=\frac{1}{V^{j}} \sum_{l=1 . . V^{j}} v_{l}^{j},
$$

that can be rewritten in

$$
\sum_{k=1 . . V^{j-1}} v_{k}^{j}+\gamma^{j} \sum_{k=1 . . V^{j-1}} \frac{1}{K_{k}^{j}} \sum_{i=1 . . K_{k}^{j}} c_{S_{k}^{j}(i)}^{j} \cong \frac{1}{4} \sum_{l=1 . . V^{j}} v_{l}^{j},
$$

We finally obtain the optimal $\gamma_{j}$ for each resolution $j$ :

$$
\gamma \cong \frac{\frac{1}{4} \sum_{l=1 . . V^{j}} v_{l}^{j}-\sum_{k=1 . . V^{j-1}} v_{k}^{j}}{\sum_{k=1 . . V^{j-1}} \frac{1}{K_{k}^{j}} \sum_{i=1 . . K_{k}^{j}} c_{S_{k}^{j}(i)}}
$$

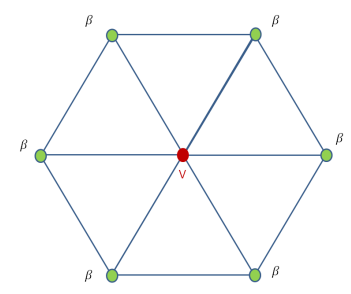

Fig. 5. Stencil of the Butterfly-based update step $U$.

\section{EXPERIMENTAL RESULTS}

To prove the interest of our optimization technique in terms of compression, we compare our optimized lifted Butterfly scheme with the classical one. We apply both schemes to three models: VASE LION, BIMBA and JOAN OF ARC. As coding scheme, we use the zerotree-based coder developed by Khodakovsky et al. for semiregular meshes in [2]. Figures 6, 7 and 8 show the PSNR (based on the Haussdorff distance [18] between a semi-regular mesh and its coded version) curves for those models when using the optimized and the classical lifted Butterfly scheme. We observe that we obtain a gain up to $2 \mathrm{~dB}$ when using our optimized scheme.

Table 1, 2 and 3 show the entropy of the obtained wavelet coefficients when using the optimized and the classical lifted Butterfly scheme. We observe that our optimization allows a significant reduction of the entropy of the sets of coefficients. As expected, this leads to better compression performances as explained previously.

\section{CONCLUSION AND FUTURE WORKS}

We described in this paper, a new method to optimize the butterflybased lifting scheme for semi-regular meshes. Our optimization consists in computing new weights for the stencils of the predictor $(P)$ and update $(U)$ operators. This optimization is done at each level of decomposition during the analysis, producing $P$ and $U$ optimized

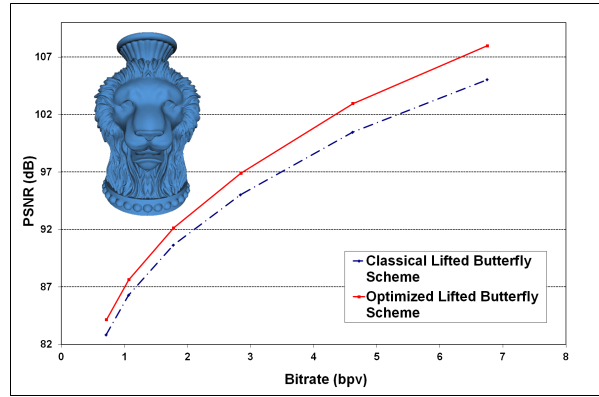

Fig. 6. PSNR curves for VASE LION.

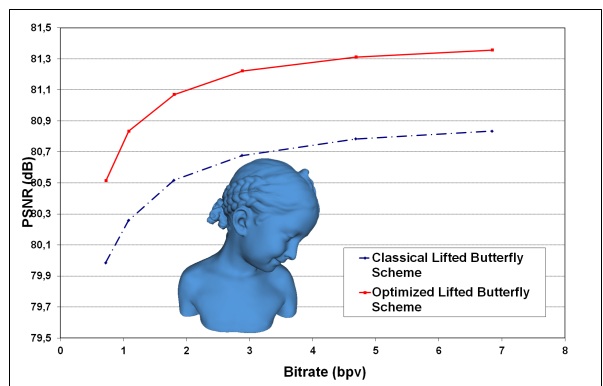

Fig. 7. PSNR curves for BIMBA.

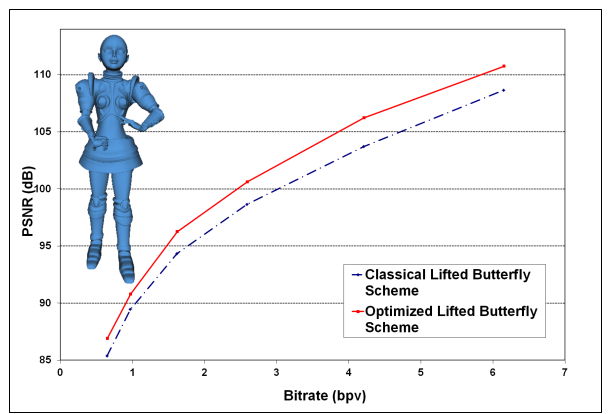

Fig. 8. PSNR curves for JOAN OF ARC.

for each level of resolution. Experimental results show that we obtain better compression performance (a gain up to $2 \mathrm{~dB}$ ) when using our optimized lifted Butterfly scheme compared to the classical one. Future works will concern:

- computing a new update operator in order to preserve higher order moments;

- a region-based approach that will partition the input mesh in function of the geometrical details, and then computing at each level of resolution different predictor operators for each partition.

This should further improve the compression performances of our approach.

\section{ACKNOWLEDGMENTS}

The original (irregular mesh) VASE LION model is courtesy of "SenSable Technologies". The original JOAN OF ARC model is 
courtesy of "I3S Laboratory". The original BIMBA model is courtesy of IMATI and INRIA. All models were remeshed with Trireme remesher [19].

\begin{tabular}{|c|c|c|c|c|c|c|}
\cline { 2 - 7 } \multicolumn{1}{c|}{} & \multicolumn{3}{c|}{ Classical } & \multicolumn{3}{c|}{ Optimized } \\
\hline res & $x$ & $y$ & $z$ & $x$ & $y$ & $z$ \\
\hline 1 & 3.9882 & 4.1804 & 4.3975 & 3.9050 & 3.9372 & 4.4277 \\
\hline 2 & 2.8992 & 2.8880 & 3.5541 & 2.8034 & 2.9219 & 3.4010 \\
\hline 3 & 1.6817 & 1.6671 & 2.6004 & 1.6364 & 1.6211 & 2.5024 \\
\hline 4 & 0.5673 & 0.5344 & 1.1387 & 0.5240 & 0.4893 & 1.1324 \\
\hline 5 & 0.0784 & 0.0726 & 0.2216 & 0.0664 & 0.0640 & 0.2231 \\
\hline 6 & 0.0016 & 0.0010 & 0.0132 & 0.0012 & 0.0008 & 0.0138 \\
\hline
\end{tabular}

Table 1. Comparison between the entropy of the wavelet coefficients obtained with a classical lifted Butterfly scheme and the entropy of the wavelet coefficients obtained with the optimized lifted Butterfly scheme for the VASE LION. The value res represents the different levels of resolution.

\begin{tabular}{|c|c|c|c|c|c|c|}
\cline { 2 - 7 } \multicolumn{1}{c|}{} & \multicolumn{3}{c|}{ Classical } & \multicolumn{3}{c|}{ Optimized } \\
\hline res & $x$ & $y$ & $z$ & $x$ & $y$ & $z$ \\
\hline 1 & 4.0197 & 4.2339 & 4.1841 & 3.7708 & 4.0243 & 4.0230 \\
\hline 2 & 3.2305 & 3.2851 & 4.0592 & 3.0672 & 3.3452 & 3.7501 \\
\hline 3 & 1.9456 & 1.9339 & 3.1158 & 1.8110 & 1.9287 & 2.9047 \\
\hline 4 & 0.7015 & 0.6197 & 1.4781 & 0.6829 & 0.5922 & 1.3781 \\
\hline 5 & 0.1270 & 0.0938 & 0.3712 & 0.1178 & 0.0812 & 0.3320 \\
\hline 6 & 0.0047 & 0.0033 & 0.0385 & 0.0029 & 0.0025 & 0.0330 \\
\hline 7 & 0.0000 & 0.0000 & 0.0008 & 0.0000 & 0.0000 & 0.0007 \\
\hline
\end{tabular}

Table 2. Comparison between the entropy of the wavelet coefficients obtained with a classical lifted Butterfly scheme and the entropy of the wavelet coefficients obtained with the optimized lifted Butterfly scheme for the BIMBA. The value res represents the different levels of resolution.

\begin{tabular}{|c|c|c|c|c|c|c|}
\cline { 2 - 7 } \multicolumn{1}{c|}{} & \multicolumn{3}{c|}{ Classical } & \multicolumn{3}{c|}{ Optimized } \\
\hline res & $x$ & $y$ & $z$ & $x$ & $y$ & $z$ \\
\hline 1 & 2.2310 & 2.6347 & 2.3789 & 2.3276 & 2.7351 & 2.3788 \\
\hline 2 & 4.3037 & 4.6194 & 4.7379 & 3.9109 & 4.3034 & 4.2994 \\
\hline 3 & 3.4381 & 3.6332 & 4.0224 & 3.3984 & 3.6322 & 3.4908 \\
\hline 4 & 2.1756 & 2.1331 & 2.3627 & 2.1461 & 2.1493 & 2.2297 \\
\hline 5 & 0.8379 & 0.8122 & 0.9690 & 0.8358 & 0.8145 & 0.9546 \\
\hline
\end{tabular}

Table 3. Comparison between the entropy of the wavelet coefficients obtained with a classical lifted Butterfly scheme and the entropy of the wavelet coefficients obtained with the optimized lifted Butterfly scheme for the JOAN OF ARC. The value res represents the different levels of resolution.

\section{REFERENCES}

[1] P. Jingliang, K. Chang-Su, and C.-C. Jay Kuo, "Technologies for 3d mesh compression: A survey," Journal of Visual Communication and Image Representation, vol. 16, pp. 688-733, December 2005

[2] A. Khodakovsky, P. Schröder, and W. Sweldens, "Progressive geometry compression," in SIGGRAPH'OO Proceedings of the 27th annual conference on Computer graphics and interactive techniques. 2000, pp. 271-278, ACM Press/Addison-Wesley Publishing Co.
[3] A. Khodakovsky and I. Guskov, "Compression of normal meshes," in Geometric Modeling for Scientific Visualization. 2003, pp. 189-206, Springer-Verlag.

[4] F. Payan and M. Antonini, "An efficient bit allocation for compressing normal meshes with an error-driven quantization," Computer Aided Geometry Design, vol. 22, no. 5, pp. 466-486, 2005.

[5] S. Dahlke, W. Dahmen, I. Weinreich, and E. Schmitt, "Multiresolution analysis and wavelets on s2 and s3," Numerical functional analysis and optimization, vol. 16, no. 1,2, pp. 1941, 1995.

[6] W. Freeden and U. Windheuser, "Spherical wavelet transform and its discretization," Advances in Computational Mathematics, vol. 5, no. 1, pp. 51-94, 1996.

[7] W. Sweldens, "The lifting scheme: A custom-design construction of biorthogonal wavelets," Applied and Computational Harmonic Analysis, vol. 3, no. 2, pp. 186-200, 1996.

[8] M. Lounsbery, T. DeRose, and J. Warren, "Multiresolution analysis for surfaces of arbitrary topological type," ACM Transactions on Graphics, vol. 16, no. 1, pp. 34-73, 1997.

[9] J. Kovacevic and W. Sweldens, "Wavelet families of increasing order in arbitrary dimensions," IEEE Transactions on Image Processing, vol. 9, no. 3, pp. 480-496, march 2000.

[10] M. Bertram, "Biorthogonal loop-subdivision wavelets," Computing, vol. 72, no. 1-2, pp. $29-39,2004$.

[11] D. Li, K. Qin, and H. Sun, "Unlifted loop subdivision wavelets," Pacific Conference on Computer Graphics and Applications, vol. 0, pp. 25-33, 2004.

[12] S. Valette and R. Prost, "Wavelet based multiresolution analysis of irregular surface meshes," IEEE Transactions on Visualization and Computer Graphics, vol. 10, no. 2, march/april 2004.

[13] D. Zorin, P. Schröder, and W. Sweldens, "Interpolating subdivision for meshes with arbitrary topology," in Proceedings of SIGGRAPH 96, Semptember 1996, pp. 189-192.

[14] F. Payan and M. Antonini, "Mean square error approximation for wavelet-based semiregular mesh compression," Transactions on Visualization and Computer Graphics (TVCG), vol. 12, July/August 2006.

[15] W. Sweldens, "The lifting scheme: A construction of second generation wavelets," SIAM Journal on Mathematical Analysis, vol. 29, no. 2, pp. 511-546, 1998.

[16] D. Donoho and Y. Tsaig, "Fast solution of $1_{1}$-norm minimization problems when the solution may be sparse," IEEE Transactions on Information Theory, vol. 54, no. 11, pp. 4789-4812, 2008.

[17] S. Bischoff and L. Kobbelt, "Teaching meshes, subdivision and multiresolution techniques," Computer-Aided Design, vol. 36, no. 14, pp. 1483-1500, 2004.

[18] N. Aspert, D. Santa-Cruz, and T. Ebrahimi, "Mesh: Measuring errors between surfaces using the hausdorff distance," in IEEE International Conference in Multimedia and Expo (ICME), 2002.

[19] Igor Guskov, "Manifold-based approach to semi-regular remeshing," Graph. Models, vol. 69, no. 1, pp. 1-18, 2007. 